AN. MED. INTERNA (Madrid) Vol. 18, N. $^{\circ} 5$, pp. $265-268,2001$

\title{
Intoxicación aguda acuosa como complicación de una urografía intravenosa
}

\author{
T. LÓPEZ DEL VAL, D. DEL OLMO, J. DIAGO, V. ALCAZAR, \\ E. HERNÁNDEZ, C. VÁZQUEZ
}

Sección de Endocrinología y Nutrición. Hospital Severo Ochoa. Leganés. (Madrid)

\author{
ACUTE WATER INTOXICATION AS A COMPLICATION \\ OF INTRAVENOUS UROGRAPHY
}

\section{RESUMEN}

En circunstancias fisiológicas la osmolalidad plasmática se regula por dos mecanismos complementarias: la hormona antidiurética (ADH) y la sed. Cuando se produce una disminución de la osmolalidad por incremento del agua en el organismo se inhibe la sensación de sed y la secreción de ADH, siendo el exceso de agua eliminado por el riñón. Cuando aumenta la osmolalidad se libera ADH, que disminuye la pérdida de agua por el riñón, y aparece la sed, produciendo ingreso de agua por vía digestiva.

La intoxicación acuosa aguda es excepcional en personas sin patología psiquiátrica crónica. Presentamos un caso de intoxicación acuosa aguda durante la realización de una urografía intravenosa. La intoxicación se produjo por una ingesta acuosa excesiva junto con la liberación de $\mathrm{ADH}$ por estrés psicológico, que impidió la eliminación de agua por el riñón. De los 9 casos semejantes previamente descritos, la mayoría han sido en mujeres y en el contexto de la preparación de pruebas diagnósticas.

PALABRAS CLAVE: Hiponatremia. Intoxicación acuosa aguda. Urografía intravenosa. Regulación de la osmolalidad.

\begin{abstract}
Physiologically, two complememtary mechanisms regulate plasma osmolality: antduretic hormone $A D H$ ) and thirst. $A D H$ release s supres sed, thirst s inhbted and renal water loss occurs when plasma osmolality belows a threshold level. Yhe rse in plasma osmolalty causes ADH relea se, stimulation of thirst and water intake.

Acute water intoxication is exceptional in patients without a chronic psychiatric disease. Herein, we describe a case of acute water intoxica tion in a prevously healthy patient, after makng an intravenous uro graphy. The excessive water intake and the impossibility of renal water loss because of streee-induced ADH release originated $t$. Only nine cases have been previously described; almost they all were women preparing for diagnostic procedures.
\end{abstract}

KEY WORDS: Hyponatremia. Acute water intoxication. Intravenous urography. Plasma osmolality regulation.

López del Val T, Del Olmo D, Diago J, Alcazar V, Hernández E, Vázquez C. Intoxicación aguda acuosa como complicación de una urografía intravenosa. An Med Interna (Madrid) 2001; 18: 265-268.

\section{INTRODUCCION}

La urografía intravenosa es una prueba frecuentemente utilizada para el diagnóstico de patología nefrourológica. Se trata de una exploración con escasos efectos secundarios, si excluímos las reacciones al contraste yodado. En la última década se han descrito intoxicaciones acuosas graves, en pacientes sometidos a pruebas diagnósticas que precisan la replección completa de la vejiga, como ocurre, por ejemplo, con la ecografía pélvica y con la urografía intravenosa.

Presentamos un caso de intoxicación acuosa aguda durante la realización de una urografía intravenosa. Se revisan los casos clínicos semejantes comunicados en la literatura (Medline 1966-1998) y se discuten la fisiopatología, los factores de riesgo y las medidas preventivas de este tipo de complicación.

\section{CASO APORTADO}

Mujer de 44 años de edad que acude a urgencias por estupor. Estaba diagnosticada de hipertensión arterial esencial y de hipotiroidismo postquirúrgico por bocio multinodular, en tratamiento con $20 \mathrm{mg}$ de nifedipino y $75 \mathrm{mcg}$ de LT4 diarios. La paciente no tomaba ninguna otra medicación. Durante los últimos tres meses había presentado infecciones urinarias de repetición con sintomatología ansiosa reactiva

Trabajo aceptado: 13 de Octubre de 1999

Correspondencia: T. López del Val. Sección de Endocrinología y Nutrición. Hospital Severo Ochoa. Avenida de Orellana, s/n. 28911 Leganés. Madrid. 
y anorexia, con pérdida secundaria de $2-3 \mathrm{~kg}$ de peso. Para el estudio de vías urinarias se le solicitó la realización de una UIV. La noche anterior a la prueba se administró una solución laxante osmótica y 2 enemas de limpieza. El día de la prueba, para conseguir la imagen vesical adecuada, se le indicó la necesidad de beber 3 litros de agua en 2 horas, circunstancia que le provocó un estado de ansiedad intensa. Poco tiempo después de la ingesta hídrica de los 3 litros comienzo con mareos y sensación de giro de objetos, siendo traída inmediatamente al servicio de urgencias, donde llegó en estado estuporoso.

En la exploración al ingreso la paciente tenía una TA de 120/80, Fc 96 lpm, Fr 20 pm, T 35,5. Era delgada. Estaba estuporosa, bradipsíquica, con tendencia al sueño y disminución de la capacidad de concentración. Tenía nistagmus bilateral en la mirada lateral extrema. Las pupilas eran isocóricas y normorreactivas, sin rigidez de nuca. Tenía temblor de reposo en extremidades superiores, ROT exaltados y simétricos, con clonus rotuliano bilateral inagotable y RCP en flexión bilateral. La auscultación cardiopulmonar fue normal y el abdomen sin hallazgos.

Analítica al ingreso: glucosa $116 \mathrm{mg} / \mathrm{dl}$, urea $32 \mathrm{mg} / \mathrm{dl}$, Na 118 $\mathrm{mEq} / \mathrm{l}$, K $3,4 \mathrm{mEq} / \mathrm{l}$, Hb 10,7, Hct 31,6, Leucocitos $8.260(58,8 \%$ neutrófi los, 30,2\% linfocitos, 6,3\% monócitos, 1,9\% eosinófilos, 0,5\% basófilos), Plaquetas 193.000, Osmolalidad $264 \mathrm{mOsm} / \mathrm{k}$ (normal 275-295 mOsmo/k), Ph 7,35, CO3H 16 mmol/1, PCO2 29mmHg (gases venosos). Cortisol sérico $42,6 \mathrm{mcg} / \mathrm{dl}$, TSH 2,68 mcU/ml, T4 libre $1,20 \mathrm{ng} / \mathrm{dl}$, T3 total $0,8 \mathrm{ng} / \mathrm{ml}$. Orina: sistemático y sedimento normal, glucosa negativa, cetonuria $150 \mathrm{mg} / \mathrm{dl}, \mathrm{Ph} 5$, Densidad 1015, $\mathrm{Na}$ 96,0 mEq/l, K 33,0 mEq/l, Osmolalidad 66,0 mOsm/k.

Inicialmente fue tratada con suero fisiológico $(250 \mathrm{ml}$ en las primeras 3 horas), pero dada la estabilidad de los síntomas clínicos se decidió tratamiento con restricción de líquidos exclusivamente. A las 7-8 horas del ingreso inició diuresis espontánea, emitiendo $4.220 \mathrm{ml}$ de orina en las siguientes 10 horas y desapareciendo paulatinamente las alteraciones neurológicas que motivaron su hospitalización. A las 17 horas del ingreso los valores de electrolitos eran los siguientes: $\mathrm{Na} 141,0 \mathrm{mEq} / \mathrm{l}, \mathrm{K} 4,1 \mathrm{mEq} / \mathrm{l}$, osmolalidad sangre $290,0 \mathrm{mOsm} / \mathrm{kg}$.

Bioquímica rutinaria normal) Incluyendo creatinina, albúmina, ácido úrico, proteínas totales y gases venosos.

A los 4 días del ingreso se realizó una sobrecarga hídrica. La paciente pesaba $36,9 \mathrm{Kg}$ y medía $143,6 \mathrm{~cm}$. Se le administraron 750 cc de agua, vía oral, durante 10-15 minutos. Antes de iniciar el test tenía una osmolalidad de 283,0 mOsm/ $/ \mathrm{kg}$, Na 141,0 mEq/l, K 4,2 $\mathrm{mEq} / \mathrm{l}$. La TSH era de 3,19 $\mathrm{mcU} / \mathrm{ml}$ y el cortisol sérico de 13,6 $\mathrm{mcg} / \mathrm{dl}$. Osmolalidad en orina 465,0 mOsm/kg; a las 2 horas, la diuresis había sido de $830 \mathrm{ml}$ y presentaba la siguiente bioquímica: Osm sangre 276,0, Na 139,4, K 3,8, Osm orina 101,0. Su TA permaneció estable sin tto farmacológico a lo largo de todo su lngreso.
Durante los siguientes 2 años la paciente ha permanecido asintomática con valores de sodio, osmolalidad plasmática y cortisol basal, normales.

\section{DISCUSIÓN}

En circunstancias fisiológicas la osmolalidad plasmática se encuentra entre $275-290 \mathrm{mosmol} / \mathrm{kg}$ (1) El agua del organismo se pierde constantemente por el riñón, los pulmones, la piel y el tracto alimentario. Para mantener la homeostasis acuosa existen dos mecanismos complementarlos de aumento de agua, que se disparan cuando se inicia un ascenso en la osmolalidad: a) la $\mathrm{ADH}$ (hormona antidiurética) hipotalámica que empieza su liberación a los $284 \mathrm{mOsm} / \mathrm{kg}$ (umbral osmótico) (2) inhibiendo la excreción de agua libre por el riñón, y b) la sensación de sed, con origen en osmorreceptores hipotalámicos, que aparece con 284-286 mOsm/kg (2), y determina ingreso de agua a través de la vía digestiva. Cuando se produce aumento de agua libre en el organismo, se inhibe completamente la secreción de $\mathrm{ADH}$ y la sed, y el riñón elimina el exceso de agua (hasta un máximo de 10-20 litros 24 oras). El pico de diuresis máxima se puede retrasar 90-120 minutos, tiempo necesario para metabolizar la $\mathrm{ADH}$ circulante existente (1).

En la patogenia del síndrome de secreción inadecuada de $\mathrm{ADH}$ (SSIADH), existen concentraciones de ADH que son inapropiadamente altas para el grado de osmolalidad plasmática. De acuerdo con los criterios diagnósticos del SSIADH (Fig. 1), nuestra paciente tuvo un SSIADH temporal con resolución espontánea.

La determinación de ADH, que no resulta imprescindible para el diagnóstico (3-4), hubiera sido interesante en el contexto del caso, pero no se pudo realizar.

En la tabla I se describen las causas de SSIADH. Existen, además, causas temporales de SSIADH (4), es decir, "factores de riesgo" que estimulan la liberación puntual de $\mathrm{ADH}$, incluso en presencia de hipotonicidad plasmática, como el traumatismo quirúrgico, el dolor, la ansiedad y el estrés en general, así como los psicofármacos.

En el SSIADH se produce una intoxicación acuosa o hiponatremia de carácter crónico e instauración paulatina.

La intoxicación acuosa aguda es frecuente en pacientes con patología psiquiátrica grave (como la esquizofrenia)

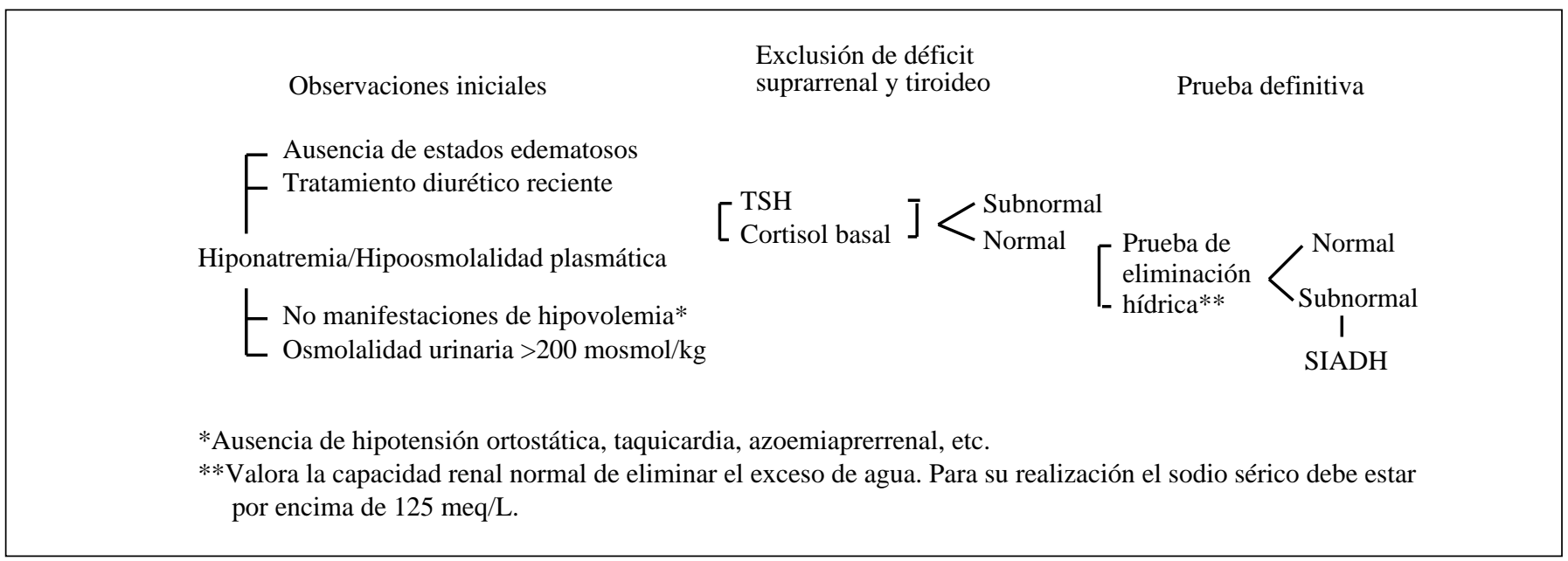

Fig. 1. Estudio diagnóstico del SSIADH. 


\section{TABLA I}

\section{CAUSAS DE SSIADH}

\begin{tabular}{ll}
\hline Neoplasias malignas con liberación autónoma & \\
de ADHF & Atrofia cerebral \\
Carcinoma pulmonar de células pequeñas & Encefalitis aguda \\
Carcinoma broncogénico & M eningitis tuberculosa \\
Linfoma,linfomalinfocítico & M eningitis purulenta \\
enfermedad de Hodgkin & Síndrome de Guillain-Barré \\
Carcinoma de duodeno & Lupus eritematoso \\
Timoma & Porfiria aguda intermitente \\
& \\
Enfermedades pulmonares no malignas & Fármacos \\
Tuberculosis & Clorpropamida \\
Absceso pulmonar & Clofibrato \\
Neumonía & Carbamacepina \\
Neumonitis viral & Ciclofosfamida \\
Empiema & Vincristina \\
Enfermedad pulmonar obstructiva & Vinblastina \\
crónica & Beta-adrenérgicos \\
& Oxitocina \\
Trombosis cerebrovascular & Anestésicos generales \\
Hematoma subdural & Narcóticos \\
& Antidepresivos tricíclicos \\
& \\
\hline
\end{tabular}

Fuente: Hatrison. Principios de M edicina Interna. 14ạ edición (3). con polidipsia crónica y osmolalidades en el límite bajo, con supresión permanente de $\mathrm{ADH}$, que hacen hiponatremia aguda por otro factor de riesgo imprescindible: la ingestión masiva de agua en un momento determinado. Existen numerosas observaciones clínicas de este tipo de casos (5-8).

La intoxicación acuosa aguda en adultos sin patología psiquiátrica ni administración parenteral excesiva de líquidos hipotónicos es excepcional. Hemos encontrado 9 casos descritos en la literatura (Tabla II).

El mecanismo común parece ser la ingesta acuosa excesiva sin solutos acompañantes junto a un SSIADH temporal, en este caso producido por ansiedad. En nuestra paciente debieron ser además causas coadyuvantes la pérdida reciente de peso con disminución de solutos urinarios $(1,7)$, y cierto grado de hipovolemia previa a la prueba por la administración de la solución evacuante.

Como en el resto de los casos, en nuestra paciente el restablecimiento de la osmolalidad normal fue bastante precoz (16-18 horas); situada en condiciones de reposo y tranquilidad se inhibió correctamente la $\mathrm{ADH}$, y se inició una diuresis masiva. En el SSIADH la corrección de la osmolalidad por medio de restricción acuosa se prolonga durante días, ya que la secreción de ADH permanece elevada limitando la capacidad renal de eliminar el exceso de agua.

De los 10 casos resumidos en la tabla II, conviene destacar que la intoxicación acuosa aguda es más frecuente en mueres ( 8 casos), posiblemente por su menor superficie corporal, y que en 6 casos se debió a la preparación de pruebas diagnósticas.

Por otra parte, excepto en los dos casos que fallecieron en el momento agudo, el restablecimiento clínico fue completo sin secuelas neurológicas.

Como conclusión, es necesario insistir en que no se deben aconsejar dosis masivas de agua en la preparación de pruebas diagnósticas. Una cifra de $20-30 \mathrm{ml} / \mathrm{kg}$ de peso a beber en $1-2$ horas es una cantidad segura y suficiente para el adecuado relleno vesical en este tipo de exploraciones.

TABLA II

CASOS DE INTOXICACIÓN ACUOSA AGUDA EN ADULTOS SIN ENFERM EDADES NEUROPSIQUIÁTRICAS

\begin{tabular}{|c|c|c|c|c|c|c|}
\hline $\begin{array}{l}\text { Paciente } \\
\text { Sexo/ edad }\end{array}$ & $\begin{array}{c}\text { Ingesta de agua } \\
\text { Volumen (L) Duración (mn) }\end{array}$ & Motivo & $\begin{array}{l}\text { Sodio } \\
\mathrm{mEq} / \mathrm{l}\end{array}$ & $\begin{array}{c}\mathrm{m} 0 \mathrm{sm} / \mathrm{kg} \\
\text { Sangre/orina }\end{array}$ & $\begin{array}{l}\text { Restablecimiento } \\
\text { (en horas) }\end{array}$ & Fuente \\
\hline $1 / M / 28$ & $6-8 / 300$ & Estrechamiento & 117 & $252 / 277$ & 19 & Friedman (9) \\
\hline $2 / F / 40$ & $15 / 120$ & Ingesta de lejía* & 111 & no aportado & Falleció & Anastassiades (10) \\
\hline $3 / F / 79$ & $1,5-2 /--$ & Eco pélvica & 122 & no aportado & 24 & Chistenson (11) \\
\hline $4 / F / 21$ & $6 /--$ & Eco pélvica & 127 & no aportado & 24 & Kott (12) \\
\hline $5 / F / 64$ & $1,35 / 120$ & Eco pélvica & 123 & no aportado & 14 & Lipsk (13) \\
\hline $6 / F / 80$ & $4 /--$ & Eco abdominal & 119 & $285 /$ no aportado & 24 & Shapira (14) \\
\hline $7 / F / 40$ & $3 / 180$ & Testdeoina** & 121 & $243 / 203$ & 24 & Klonoff (15) \\
\hline $8 / M / 19$ & $--/--$ & Ejercicio físico*** & 115 & $240 / 400$ & 36 & Putterman (16) \\
\hline $9 / F / 27$ & $--/ 180-240$ & Gastro enteritis**** & 106 & no aportado & Falleció & Sjöblom (17) \\
\hline $10 / F / 44$ & $3 / 120$ & Urografía IV & 118,4 & $264 / 636$ & 17 & Presente estudio \\
\hline
\end{tabular}

*Ingesta masiva de agua tras ingesta de lejía. **M uestra de orina para prueba dignóstica. *** Ingesta masiva de agua tras ejercicio físico intenso. $* * * *$ Ingesta masiva de agua tras gastroenteritis. 


\section{Bibliografía}

1. Rose BD, editor Clinical physiology of acid-base and electrolyte disorders. 4 ed. New York: McGraw-Hill, Inc, 1994.

2. McKenna K, Thompson C. Osmoregulation in clinical disorders of thirst appreciaton. Clinical Endocrinology 1998; 49: 139-152.

3. Moses AM, Streeten DH. Trastornos de la neurohipófisis. En: Harrison. Principios de Medicina Interna. Fauci, Braunwald, Isselbacher, Wilson, Martin, Kasper, Hauser, Longo, editores. 14 ed. McGraw-Hill Interamericana de España. Madrid 1998; 2276-2286.

4. Aron CD, Findling JW, Tyrrel JB. Hypothalamus and Pituitary. En: Basic and Clinical Endocrinology. Greenspan, Strewler, editores. 5 ed. Appleton and Lange. Stamford 1997; 95-156.

5. Hanihara T, Amagai 1, Hagimoto H, Makimoto Y. Hypouricemia in chronic schizophrenic patients with polydipsia and hyponatremia. J Clin Psychiatry 1997; 58: 256-260.

6. Ellinas PA, Rosner F, Jaume LC. Symptomatic hyponatremia asociated with psychosis, medications, and smoking. J Natl Med Assoc 1993; 85: 135-141.

7. Tanneau R, Pennec YL, Morin JF, Codet JP, Bourbigot B, Garre M, Le Menn G. Salt wastage, plasma volume contraction and hypokalemic paralysis in selfinduced water intoxication. Nephron 1993; 64: 570-575.

8. Korets A, Ori Y, Floro S, Osh-Tov E, Chagnac A, Weinstein T, et al.
Case report: Severe hyponatremia after water intoxication: A potential cause of rhabdomyolysis. Am J Med 1996; 12: 92-94.

9. Friedman E, Hanany J, Halkin H. Unsuspected urethral stricture presenting as acute water Intoxlcatlon J Urol 198; 130: 566.

10. Anastassiades E, Wilson R, Stewart JSW, Perkin GD. Fatal brain oedema due to accidental water intoxication. BMJ 1983; 287: 1 81-1182.

11. Christenson LL, Scott D. Acute water intoxication following pelvic ultrasound examination. Postgrad 11ed 1985; 77: 161-162.

12. Kott E, Marcus Y. Acute brain edema due to water loading in a young woman. Eur Neurol 1985; 24: 221-224.

13. Lipsky S, Zabak DL. Hyponatremia resulting from pelvic ultrasound preparation. J Fam Pract. 1987; 25: 216-217.

14. Shapira 1, Isakof A, Almog C. Hyponatremia as the result of preparation for abdominal ultrasound examination. JCU 1988; 16: 61-62.

15. Klonoff DC, Jurow AH. Acute water intoxication as a complication of urine drug testing in the workplace. JAr1A 1991; 265: 84-85.

16. Putterman C, Levy L, Rubinger D. Transient exercise-induced water intoxication and rhabdomyolysis. Am J Kidney Dis 199; 21: 206-209.

17. Sjoblom E, Hojer J, Ludwigs U, Pirskanen R. Fatal hyponatraemic brain oedema due to common gastroenteritis with accidental water intoxication. Intensive Care Med 1997; 2: 348-50. 